\title{
Introduction to the Minitrack "Modeling and Decision Making in Manufacturing and Logistics in the Age of Industry 4.0"
}

\author{
Tobias Reggelin \\ Otto von Guericke \\ University Magdeburg \\ tobias.reggelin@ovgu.de
}

\author{
Stefan Galka \\ Ostbayerische Technische \\ Hochschule Regensburg \\ stefan.galka@oth-regensbu \\ rg.de
}

\author{
Dmitry Ivanov \\ Berlin School of \\ Economics and Law \\ divanov@hwr-berlin.de
}

\author{
Sebastian Lang \\ Otto von Guericke \\ University Magdeburg \\ sebastian.lang@ovgu.de
}

\begin{abstract}
The minitrack contains contributions with a focus on modeling and decision making in manufacturing and logistics in the context of Industry 4.0. Cyber-physical systems integrate the real world and the virtual world. For example, customer decisions and supply disruptions which directly influence manufacturing and logistics systems require immediate decisions in terms of planning and controlling the manufacturing and logistics systems. In order to immediately respond to these dynamics, models must have the capability to support decision making in manufacturing as well as in internal and external logistics in real-time. Two of the Mintrack papers describe concepts and models for digital twins. One paper provides an adaptive scheduling framework for solving multi-objective hybrid flow shop scheduling problems and one paper investigates matrix production systems and with their requirements and impacts on logistics planning.
\end{abstract}

\section{Digital Twins for production and logistics}

The term Digital Twin is used in many different ways these days. In most cases, this means the virtual representation of a product, process or system. The research institute Gartner assumes that half of all large industrial companies will use a form of a digital twin by the year 2021 and that this will increase their effectiveness by 10 percent [1]. Kauke, Galka, and Fottner [2] describe a concept for a digital twin used for controlling and monitoring of an order picking system. The described approach in the paper aims at supporting the operational control, e.g. Human resource dimension or job assignment. Both the architectural structure and the functions, e.g. the simulation, are described in detail. The paper clarified that Digital Twins cannot be considered separately from Big Data and Data Analytics, since a large amount of data has to be prepared for Digital Twins. This processing represents one of the greatest challenges within the implementation of a Digital Twin. [2] If digital twins are not only representing a single warehouse system, rather an entire supply network, the data analysis and data exchange is becoming an increasingly important factor. Cirullies and Schwede [3] describe a model that enables interorganizational on-demand data exchange in supply networks. They identify key barriers for inter- and intra organizational data exchange, deduce requirements out of these barriers and develop a concept, which fulfills these requirements to enable real-time data exchange and create a digital twin for supply networks. A concept is developed, which applies Industrial Data Space Technology to overcome these barriers for data exchange in Supply Networks. [3] For the development of digital twins, knowledge in many different areas is necessary, e.g. simulation, data analysis, interfaces/data exchange and system knowledge (of the real system).

\section{Production scheduling in the age of Industy 4.0}

Recent trends in production and logistics, for instance decreasing production leat times, broader production programs or growing uncertainties in supply chains, motivate the investigation of new production scheduling methods, which are able to compute solutions of high quality in very short time. Against this background, Nahhas, Krist, and Turowski [4] present an adaptive scheduling framework for 
hybrid flow shop scheduling problems. The authors pursue a hyper-heuristical approach. In particular, a genetic algorithm searches for a set of heuristics that are successively applied at certain points in time during the production. Thus, the authors combine both, the light execution time of heuristics as well as the robustness and solution quality of metaheuristics to achieve high-quality results within a few minutes. The framework is evaluated on case study from the industry - a 4-stage hybrid flow shop with sequence- and family-dependent setup times.

\section{Matrix Production Systems}

Schmidtke, Rettmann, and Behrendt [5] describe the requirements for flexible, dynamic routing and self-organizing resources in material supply for matrix production systems. They provide a generic conceptual model for matrix production which they use for further analysis at the example of a reference scenario from the automotive industry. The authors use material flow simulation for their analysis. The paper analyzes the impacts of decentralized material supply concepts on logistics planning and the structural differences compared to state of the art flow production.

\section{References}

[1] C. Pettey, "Prepare for the impact of digital twins," 2017.

[2] D. Kauke, S. Galka, and J. Fottner (2021). Digital Twins in Order Picking Systems for Operational Decision Support. In: Proceedings of the 54th Hawaii International Conference on System Sciences.

[3] J. Cirullies and C. Schwede (2021). On-demand Shared Digital Twins - An Information Architectural Model to Create Transparency in Collaborative Supply Networks. In: Proceedings of the 54th Hawaii International Conference on System Sciences.

[4] A. Nahhas, M. Krist, and K. Turowski (2021). An adaptive scheduling framework for solving multi-objective hybrid flow shop scheduling problems. In: Proceedings of the 54th Hawaii International Conference on System Sciences.

[5] N. Schmidtke, A. Rettmann, and F. Behrendt (2021). Matrix Production Systems - Requirements and Influences on Logistics Planning for Decentralized Production Structures. In: Proceedings of the 54th Hawaii International Conference on System Sciences. 